\title{
Nanostructured ZnO for Electrochemical Biosensors
}

\author{
Jaime E. Ramirez-Vick*
}

Engineering Science \& Materials Department, University of Puerto Rico, Mayaguez, PR 00681, USA

\section{Editorial}

Biosensors have become important and practical tools in pathogen detection, molecular diagnostics, environmental monitoring, food safety control, and homeland security. Of these, electrochemical biosensors are of particular interest due to several combined advantages such as low cost, ease of operation, and miniaturization capability. A current goal for these types of biosensors is of becoming point-ofcare diagnostic devices. Much effort has been put into improving key performance parameters, such as sensitivity, specificity, recognition rates, and multiplexing capabilities for parallel recognition, to allow this possibility.

Over the last fifteen years efforts have focused on the use of nanotechnology to develop nanostructured materials (e.g., as nanowires, nanotubes, nanoparticles and nanorods) as biomolecule immobilizing matrices to improve electrochemical detection [1]. Nanoscale structures like these offer many unique features and show great promise for faster response and higher sensitivity at the device interface than offered by planar sensor configurations. Their nanometer dimensions, being in the scale of the target analyte, show an increased sensing surface and strong binding properties, thus allowing a higher sensitivity. The interest in developing these nanostructures for biosensing applications has resulted from the development of new synthesis methods and improved characterization techniques, allowing for new functionalities to be created.

Recently, metal oxide nanomaterials, based on metals such as zinc, tin, copper, titanium, and nickel, has been the focus of many efforts in the development of improved electrochemical biosensors [2]. Of these, $\mathrm{ZnO}$ has attracted much attention because of its unique properties, suitable for biosensing applications. For instance, $\mathrm{ZnO}$ is a well-known n-type non-silica direct wide band gap semiconductor from group II-VI, with band gap energy of $3.37 \mathrm{eV}$ and a large excitonic binding energy of $60 \mathrm{meV}$ at room temperature. Its direct wide band gap makes it a good candidate for optoelectronic applications and its large exciton energy helps to employ the excitonic recombination process as a lasing mechanism. $\mathrm{ZnO}$ is a polar borderline semiconductor material with two crystallographic planes having opposite polarity and different surface relaxation energies that lead to a higher growth rate along the c-axis, e.g., formation of nanorods like vertical structures. It exists in a wurtzite crystal structure, at ambient temperature and pressure, with a hexagonal lattice, having two interconnecting sub-lattices of $\mathrm{Zn}^{2+}$ and $\mathrm{O}^{2-}$, with the zinc ion surrounded by tetrahedral oxygen ions, and vice versa. This tetrahedral coordination gives rise to a polar symmetry along the hexagonal axis, which is responsible for a number of physical and chemical properties of $\mathrm{ZnO}$, including piezoelectricity and spontaneous polarization. The large piezoelectric coefficient of $\mathrm{ZnO}$ allows the development of surface acoustic wave devices that can operate at higher frequencies. The variation in electrical properties such as conductivity is dictated by the presence of oxygen vacancies, a shallow interstitial zinc donor, hydrogen donor impurity and other donor type point defects. Moreover, the $\mathrm{ZnO}$ near-surface region can be highly conductive, due to $\mathrm{H}$ donors in this region and a large density of near-surface electrons. The high electron communication feature, biocompatibility, and chemical stability with high isoelectric point $(\sim 9)$ make $\mathrm{ZnO}$ an attractive matrix for biosensor applications.

Researchers have reported a myriad of $\mathrm{ZnO}$ nanostructures for biosensor applications synthesized through various physical and chemical routes. These include nanowires, nanorods, nanotubes, nanocombs, nanoforks, nanofibers, nanoflakes, nano-waxberries, nanobundles, nanospheres, nanocomposites, nanotetrapods, nanoparticles, nanorod spheres, nanoflowers, and nanosheets/disks [3]. It is expected that each of these nanostructures should show variations in fundamental electrical, optical and physico-chemical properties, great promise for faster response and higher sensitivity compared to other configurations.

The polymorphic capability of $\mathrm{ZnO}$ for the synthesis of nanostructured materials offers a great potential for fundamental studies in the roles of dimensionality and size based physical properties. Nanostructured $\mathrm{ZnO}$ has proven its potential as materials for biosensing applications. The ease of fabrication using low cost processes, which can yield a wide range of nanostructures, makes $\mathrm{ZnO}$ based matrices a promising platform for low cost biosensors. Moreover, the biocompatible nature of $\mathrm{ZnO}$ and compatibility with MEMS technology will play a major role towards the use of this material in designing miniaturized, wireless and implantable biosensors. Further characterization of these nanostructured materials is essential to advance de field of electrochemical biosensors and reach the goal of a sensitive, fast and inexpensive point-of-care diagnostic device.

\section{References}

1. Berti F, Turner APF (2011) New Micro- and Nanotechnologies for Electrochemical Biosensor Development. Biosensor Nanomaterials. WileyVCH Verlag GmbH \& Co. KGaA, Weinheim, Germany.

2. Solanki PR, Kaushik A, Agrawal VV, Malhotra BD (2011) Nanostructured metal oxide-based biosensors. NPG Asia Materials 3: 17-24.

3. Palomera N, Balaguera M, Arya SK, Hernández S, Tomar MS, et al. (2011) Zinc oxide nanorods modified indium tin oxide surface for amperometric urea biosensor. J Nanosci Nanotechnol 11: 6683-6689.

*Corresponding author: Jaime E. Ramirez-Vick, Engineering Science \& Materials Department, University of Puerto Rico, Mayaguez, PR 00681, USA, Tel: (787) 832-4040 Ext.5720; E-mail: jvick@ece.uprm.edu

Received March 22, 2012; Accepted March 22, 2012; Published March 26, 2012

Citation: Ramirez-Vickl JE (2012) Nanostructured ZnO for Electrochemical Biosensors. J Biosens Bioelectron 3:e109. doi:10.4172/2155- 6210.1000e109

Copyright: (c) 2012 Ramirez-Vickl JE. This is an open-access article distributed under the terms of the Creative Commons Attribution License, which permits unrestricted use, distribution, and reproduction in any medium, provided the original author and source are credited. 